\title{
Neutrino bursts from gravitational stellar collapses with LVD
}

\author{
A. Molinario and C. Vigorito \\ On behalf of the LVD Collaboration \\ Department of Physics, University of Torino and INFN Torino, Italy
}

Received: 26 October 2010 - Accepted: 28 December 2010 - Published: 3 February 2011

\begin{abstract}
The main goal of the Large Volume Detector (LVD), in the INFN Gran Sasso National Laboratory (Italy), is the study of neutrino bursts from gravitational stellar collapses in the Milky Way. Both the detector and the data analysis procedure have been actually optimized for this purpose. Moreover the modularity of the apparatus allows to obtain a duty cycle that is very close to $100 \%$, so that the experiment is continuously monitoring the Galaxy.

The search for Supernova neutrino signal is performed online, within fixed duration time windows (20 s), and offline with variable duration time windows from few ms up to $200 \mathrm{~s}$. In both cases, LVD is able to disentangle a cluster of neutrino signals from the background fluctuations, and its sensitivity extends to the whole Galaxy.

No candidates have been detected during almost 18 years of observation ( 6013 days of lifetime): the resulting $90 \%$ c.l. upper limit to the rate of gravitational stellar collapses in the Galaxy is 0.14 events/year. Detector performances, search method and data results are here reported.
\end{abstract}

\section{Introduction}

Gravitational stellar collapses are astrophysical events of great interest. Because of the complexity of the problem, the modeling of the physical processes is still in evolution, but it is in general accepted that the role of neutrinos is critical to allow the supernova to form out of a collapse (Bethe and Wilson, 1985).

The confirmed detection of the neutrino signal from the SN 1987A, which was located in the Large Magellanic Cloud, marked the beginning of a new era in neutrino astrophysics (e.g. in Hirata et al., 1987; Bionta et al., 1987;

\section{Correspondence to: C. Vigorito} (vigorito@to.infn.it)
Alekseev et al., 1987) and, in spite of some unresolved controversies Aglietta et al. (1987) opened the way for a new method of investigation: the neutrino astronomy. Even in the lack of a complete theory of the core collapse supernova explosion the correlated neutrino emission is believed to be well established and should be detected with different active detectors at the time next event will occur within the Milky Way boundaries.

\section{The LVD detector}

The Large Volume Detector (LVD), operating since June 1992 , is a $1 \mathrm{kt}$ liquid scintillator detector whose main purpose is to monitor the Galaxy looking for neutrino bursts from gravitational stellar collapses (Aglietta et al., 1992). The detector is located underground at a depth of $1400 \mathrm{~m}$ under rock (3600 m water equivalent), in the hall A of INFN Gran Sasso National Laboratory (Italy).

LVD is an array of 840 scintillator counters, $1.5 \mathrm{~m}^{3}$ each, organized in a compact structure (dimensions $13 \times 23 \times 10 \mathrm{~m}^{3}$ ). Counters are divided in three identical towers with independent high voltage power supplies, trigger logic and data acquisition. They belong to 2 different subsets, internal (400 units) and external (440 units), as a function of their exposure to the rock radioactivity: the external counters correspond to the outer shell while the internal ones to the inner core.

The trigger logic is optimized for the detection of both products of the inverse beta decay $\bar{v}_{e} p, e^{+} n$ and is based on the coincidence of the 3 photomultipliers of each single counter. Beyond the active scintillator $(M=1 \mathrm{kton})$ the iron supporting structure of the detector $(M=0.85 \mathrm{kton})$ can also act as a passive target for neutrino and products of the interactions can be detected.

The modularity of the array allows high duty cycle performance ( $\geq 99 \%$ since 2001) as shown in Fig. 1 together with

Published by Copernicus Publications on behalf of the Arbeitsgemeinschaft Extraterrestrische Forschung e.V. 


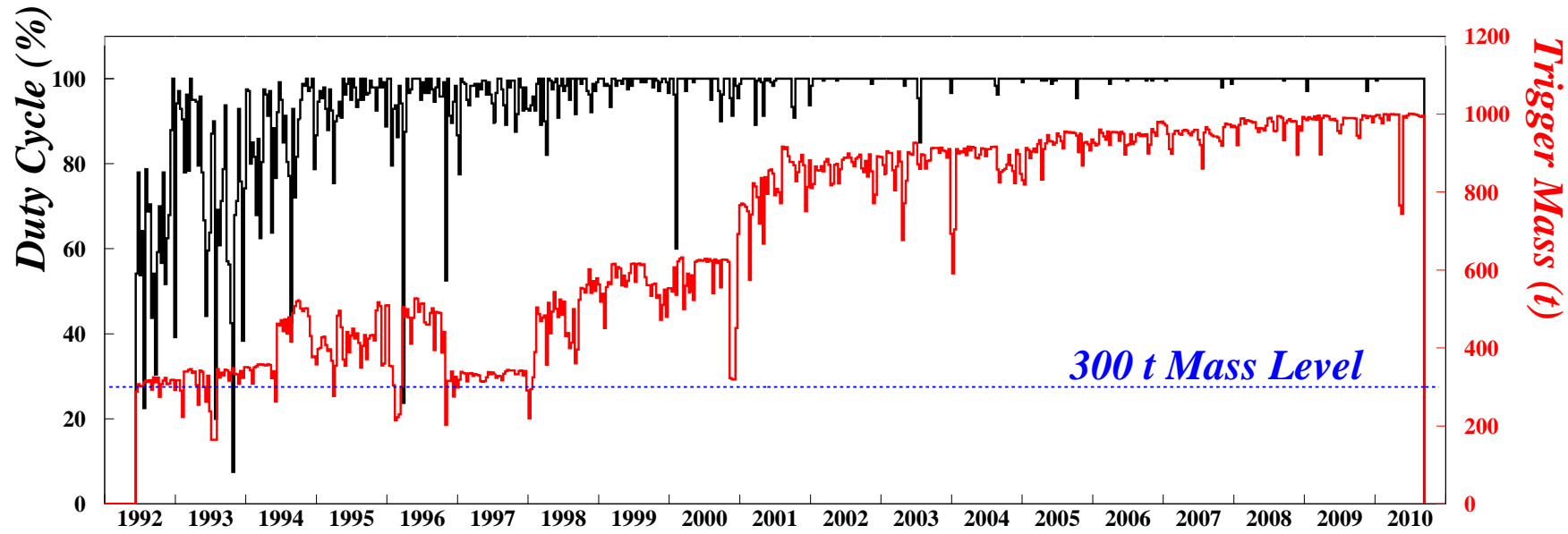

Fig. 1. Duty cycle (in black) and active mass (in red) as a function of time, updated to 31 August 2010.

the active mass profile along 18 years of data acquisition.

The main neutrino reaction in LVD is the inverse beta decay (IBD) $\bar{v}_{e} p, e^{+} n$, which gives two detectable signals: the prompt one due to the $e^{+}$(visible energy $\left.E_{\mathrm{vis}} \simeq E_{\bar{v}_{e}}-Q+m_{e}=E_{\bar{v}_{e}}-0.789 \mathrm{MeV}\right)$ followed by the signal from the $n p \rightarrow d \gamma$ capture $\left(E_{\gamma}=2.23 \mathrm{MeV}\right)$. For the detection of both products of the reaction, each PMT of a single counter is discriminated at two different thresholds resulting in two possible levels of three-fold coincidence: $\mathrm{H}$ and $\mathrm{L}$, corresponding to $\mathcal{E}_{H} \simeq 4 \mathrm{MeV}$ and $\mathcal{E}_{L} \simeq 0.5 \mathrm{MeV}$. The $\mathrm{H}$ coincidence in any counter represents the trigger condition for the module. Once a trigger has been identified, the charge and time of the three summed PMTs signals are stored in a memory buffer. All the signals satisfying the L coincidences in the same module ( 8 counters) of the trigger counter are also stored, if they occur within $1 \mathrm{~ms}$. The average neutron detection efficiency, $\epsilon_{n}$, amounts to about $50 \%$ for neutrons detected in the same counter where the positron has been observed.

Besides interactions with free protons LVD is also sensitive to charged current interactions with carbon and iron nuclei through:

- $v_{e}{ }^{12} \mathrm{C},{ }^{12} \mathrm{~N} e^{-}$, (physical threshold $E_{v_{e}}>17.3 \mathrm{MeV}$ ) observed through two signals: the prompt one due to the $e^{-}$ ( $E_{d} \simeq E_{v_{e}}-17.3 \mathrm{MeV}$ ) followed by the signal from the $\beta^{+}$ decay of ${ }^{12} \mathrm{~N}$ (mean life $\tau=15.9 \mathrm{~ms}$ );

- $\bar{v}_{e}{ }^{12} \mathrm{C},{ }^{12} \mathrm{~B} e^{+}$, (physical threshold $E_{\bar{v}_{e}}>14.4 \mathrm{MeV}$ ) observed through two signals: the prompt one due to the $e^{+}$ $\left(E_{d} \simeq E_{\bar{v}_{e}}-14.4 \mathrm{MeV}+2 m_{e} c^{2}\right)$ followed by the signal from the $\beta^{-}$decay of ${ }^{12} \mathrm{~B}$ (mean life $\tau=29.4 \mathrm{~ms}$ );

- $v_{e}{ }^{56} \mathrm{Fe},{ }^{56} \mathrm{Co} e^{-}$, where the mass difference between the nuclei is $\Delta_{m_{n}}=m_{n}^{\mathrm{Co}}-m_{n}^{\mathrm{Fe}}=4.055 \mathrm{MeV}$, and the first Co allowed state at $3.589 \mathrm{MeV}$; the efficiency for electron and gammas, also produced in the interaction, to reach the scin- tillator with energy higher than $\mathcal{E}_{H}$ has been simulated (see Agafonova et al., 2007); on average, the detectable electron energy is $E_{d} \simeq 0.45 \times E_{v}$. $-\bar{v}_{e}{ }^{56} \mathrm{Fe},{ }^{56} \mathrm{Mn} e^{+}$.

And neutral current interactions through:

- $\stackrel{(-)}{v_{\ell}}{ }^{12} \mathrm{C}, \stackrel{(-)}{v_{\ell}}{ }^{12} \mathrm{C}^{*} \quad(\ell=e, \mu, \tau), \quad$ (physical threshold $\left.E_{v}>15.1 \mathrm{MeV}\right)$, whose signature is the monochromatic photon from carbon de-excitation $\left(E_{\gamma}=15.1 \mathrm{MeV}\right)$;

- $\stackrel{(-)}{v}_{\ell}{ }^{56} \mathrm{Fe}, \stackrel{(-)}{v_{\ell}}{ }^{56} \mathrm{Fe}^{*} \quad(\ell=e, \mu, \tau), \quad$ (physical threshold $\left.E_{v}>7.6 \mathrm{MeV}\right)$

$-\stackrel{(-)}{v} \ell e^{-}, \stackrel{(-)}{v_{\ell}} e^{-}$, which yields a single signal due to the recoil electron.

\section{Search for neutrino bursts}

LVD has been taking data since June 1992 with increasing mass configurations. We consider only that periods when the sensitive mass was greater than 300 tons (see Fig. 1) enough to monitor the whole Galaxy $(D<20 \mathrm{kpc})$ as discussed in Agafonova et al. (2008). The results of the search for neutrino bursts in the period 1992-2009 have been already reported (see Vigorito et al., 2009, and references therein) and are summarized in Table 1.

The results of the analysis of the last run, since 1 May 2009 to 31 May 2010, for a total live-time of 395 days, are presented here.

The data set include all the triggers in the $7-100 \mathrm{MeV}$ energy range; a cut on crossing muons (at least two triggers in different counters within $250 \mathrm{~ns}$ window), is applied. Some quality cuts are included to avoid electronic noise that can affect data and to exclude counters with unstable counting rate. 
Table 1. LVD data runs.

\begin{tabular}{rrccc}
\hline Run & \multicolumn{1}{c}{ Start } & End & Time (days) & Mass (t) \\
\hline 1 & 6 Jun. 1992 & 31 May 1993 & 285 & 310 \\
2 & 4 Aug. 1993 & 11 Mar. 1995 & 397 & 390 \\
3 & 11 Mar. 1995 & 30 Apr. 1997 & 627 & 400 \\
4 & 30 Apr. 1997 & 15 Mar. 1999 & 685 & 415 \\
5 & 16 Mar. 1999 & 11 Dec. 2000 & 592 & 580 \\
6 & 12 Dec. 2000 & 24 Mar. 2003 & 821 & 842 \\
7 & 25 Mar. 2003 & 4 Feb. 42005 & 666 & 881 \\
8 & 4 Feb. 2005 & 31 May 2007 & 846 & 936 \\
9 & 31 May 2007 & 30 Apr. 2009 & 699 & 967 \\
10 & 1 May 2009 & 31 May 2010 & 395 & 976 \\
\hline$\Sigma$ & 6 Jun. 1992 & 31 May 2010 & 6013 & 707 \\
\hline
\end{tabular}

\subsection{The selection algorithm}

The algorithm is based on the search for clusters of $\mathrm{H}$ triggers within a fixed time window, $\Delta t=20 \mathrm{~s}$. The candidate is simply characterized by its multiplicity $\mathrm{m}$, i.e. the number of pulses detected in $\Delta t$. All the other characteristics of the cluster, detailed time structure, energy spectra, $v$ flavor content and topological distribution of signals inside the detector are left to a subsequent independent analysis. In detail, the time sequence of total duration $\mathrm{T}$, is scanned through a sliding window of duration $\Delta t=20 \mathrm{~s}$, that is, it is divided into $N=2 \cdot \frac{T}{\Delta t}-1$ intervals, each one starting in the middle of the previous one (in this way the maximum unbiased time window is $10 \mathrm{~s}$ ).

The frequency of clusters of duration $20 \mathrm{~s}$ and multiplicity $\geq m$, i.e. the imitation threshold due to background, is:

$F_{i m}\left(m, f_{b k}\right)=N \cdot \sum_{k \geq m}^{\infty} P\left(k ; \frac{20 \cdot f_{b k}}{s^{-1}}\right)$ events $\cdot \mathrm{day}^{-1}$

where: $f_{b k}$ is the background counting rate of the detector for $E \geq E_{\text {cut }} ; P\left(k, f_{b k} \Delta t\right)$ is the Poisson probability to have clusters of multiplicity $k$ if $\left(f_{b k} \Delta t\right)$ is the average background multiplicity, and $N$ is the number of trials per day.

The search for burst candidates is performed, on-line and off-line, simultaneously for two values of the energy cut: $E_{c u t}=7 \mathrm{MeV}\left(f_{b k}=0.2 \mathrm{~Hz}\right)$ and $E_{c u t}=10 \mathrm{MeV}$ ( $f_{b k}=0.03 \mathrm{~Hz}$ ), being $N^{\prime}=2 N$ the effective number of trials.

As we are able to properly describe the background behaviour in LVD, we identify as neutrino burst candidates only those clusters which would be produced by background fluctuations less than 0.01 year $^{-1}$, relaxing the threshold to 1 month $^{-1}$ when working in time coincidence with other detectors as in the SNEWS project (see Antonioli et al., 2004). Less sensitive thresholds on $F_{i m}$ (i.e. $1 \mathrm{week}^{-1}, 1 \mathrm{day}^{-1}$, 1 hour $^{-1}$ ) are used only for monitorig purpose to test the algorithm performance and stability as shown in Fig. 2. After this pure statistical selection a complete analysis of each detected cluster with $F_{i m} \leq 1 \mathrm{y}^{-1}$ is performed, to test its con-

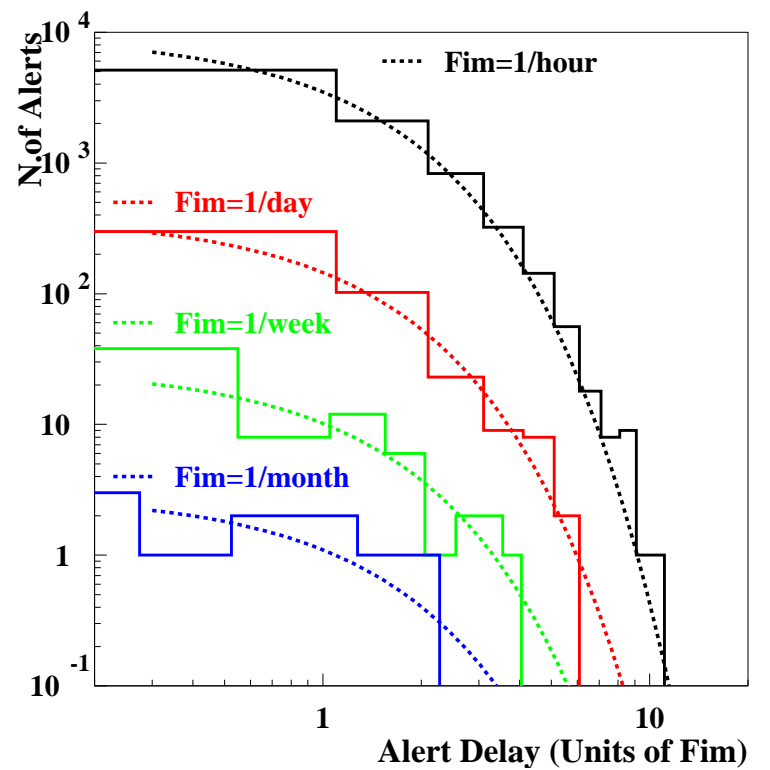

Fig. 2. Distribution of the observed time delay between alerts selected at different imitation threshold $\left(F_{i m}\right)$ from 1 hour $^{-1}$ up to 1 month $^{-1}$, compared with the expected distribution (dashed lines) at the corresponding threshold.

sistency with a $v$ burst. Additional information will come from the study of the candidate properties.

a) The energy spectrum: events from a supernova neutrino burst peak at around $20 \mathrm{MeV}$, while the events from the background reach their maximum just at the energy threshold (7 MeV).

b) The topology of the event: LVD can identify the counter where an event occurred. We expect that a neutrino burst would distribute uniformly in the detector, while events from the background favour external counters over the internal, more shielded, ones.

c) The temporal distribution of n-capture signals: a real neutrino burst would mainly interact through IBD, so we expect the temporal distribution of $\mathrm{L}$ type signals following the triggers to be exponential decaying with mean capture time $\tau=185 \mu \mathrm{s}$.

No candidates have been found in the present run analysis at level of $F_{i m} \leq 0.01 \mathrm{y}^{-1}$. Including previous negative results over a total of 6013 days the resulting $90 \%$ c.l. upper limit to the rate of gravitational stellar collapses in the Galaxy is 0.14 events / year.

\subsection{The expected sensitivity}

The selection method, as it follows from Eq. (1), defines a candidate as any cluster of $m \geq m_{\text {min }}$ signals within a window of $\Delta t=20 \mathrm{~s}$. For a known background rate, $m_{\min }-f_{b k} \Delta t$ represents the minimum number of neutrino interactions re- 


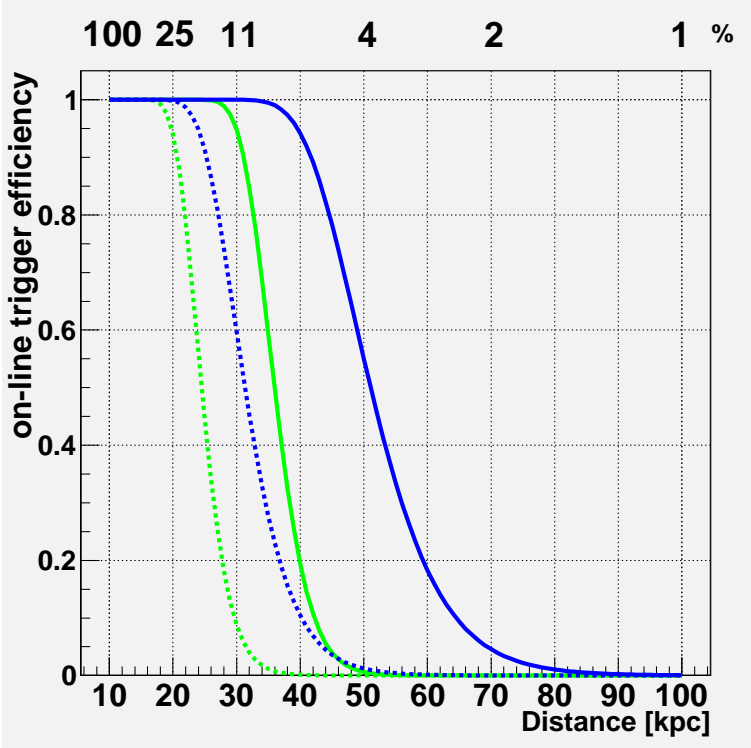

Fig. 3. On-line trigger efficiency versus distance (lower scale) and percentage of SN1987A signal at $10 \mathrm{kpc}$ (upper scale) for $E_{\text {cut }}=7-10 \mathrm{MeV}$ (light green and dark blue lines, respectively) and $\mathrm{M}=300 \mathrm{t}$ (dotted) and $1000 \mathrm{t}$ (continuous) for LVD stand alone.

quired to produce a supernova alarm at a selected $F_{i m}$ threshold (e.g. 1 year $^{-1}, 1$ month $^{-1}, \ldots$ etc.).

Assuming a model for the neutrino emission and propagation, the detector sensitivity can be expressed in terms of source distance (or emitted neutrino flux). In particular we adopt the following conservative values for the astrophysical parameters of SN1987A (Costantini et al., 2004, 2007): average $\bar{v}_{e}$ energy $\left\langle E_{\bar{v}_{e}}\right\rangle=14 \mathrm{MeV}$; total radiated energy $E_{b}=2.4 \cdot 10^{53} \mathrm{erg}$, assuming energy equipartition; average non-electron neutrino energy $10 \%$ higher than $\bar{v}_{e}$ (Keil et al., 2003), and concerning neutrino oscillations (see Agafonova et al., 2007, for a discussion), we consider normal mass hierarchy. We calculate the number of inverse beta decay signals expected from a SN1987A-like event occurring at different distances, for $E_{\text {cut }}=7$ and $E_{\text {cut }}=10 \mathrm{MeV}$. Taking into account Poisson fluctuations in the cluster multiplicity, we derive the on-line trigger efficiency as a function of the distance shown in Fig. 3 (lower scale) for LVD working stand-alone. The trigger efficiency, as a function of neutrino luminosity in terms of percentage of SN1987A one is shown in the upper scale (see details in Agafonova et al., 2008).

\section{Conclusions}

LVD has been continuously monitoring the Galaxy since 1992 in the search for neutrino bursts from gravitational stellar collapses. Its active mass has been progressively increased from $300 \mathrm{t}$ in 1992 to the final $1000 \mathrm{t}$ in 2001, always guaranteeing a sensitivity to gravitational stellar collapses up to distances $D \leq 20 \mathrm{kpc}$. The telescope duty cycle, in the last ten years, was $>99 \%$.

No burst candidate has been found over 6013 days of live-time, the resulting $90 \%$ c.l. upper limit to the rate of gravitational stellar collapses in the Galaxy $(D \leq 20 \mathrm{kpc})$ is 0.14 events / year.

Edited by: T. Suomijarvi

Reviewed by: R. Caruso and another anonymous referee

\section{References}

Agafonova, N. Yu., Aglietta, M., Antonioli, P., et al.: Study of the effect of neutrino oscillations on the supernova neutrino signal in the LVD detector, Astropart. Phys., 27, 254-270, 2007.

Agafonova, N. Yu., Aglietta, M., Antonioli, P., et al.: On-line recognition of supernova neutrino bursts in the LVD, Astropart. Phys., 28, 516-522, 2008.

Aglietta M., et al. (LSD collaboration): On the event observed in the Mont Blanc underground neutrino observatory during the occurrence of Supernova 1987a , Europhys. Lett., 3, 1315, 1987.

Aglietta M., et al. (LVD collaboration): The Most Powerful Scintillator Supernovae Detector: LVD, Il Nuovo Cimento A, 105, 1793, 1992.

Alekseev, E. N., Alekseeva, L. N., Volchenko, V. I., et al.: Possible detection of a neutrino signal on 23 February 1987 at the Baksan underground scintillation telescope of the institute of nuclear research, J. Exp. Theor. Phys. Lett., 45, 589, 1987.

Antonioli, P., Fienberg, R. T., Fleurot, F., et al.: SNEWS: the SuperNova Early Warning System, New J. Phys., 6, 114, 2004.

Bethe, H. A., and Wilson, J. R.: Revival of a stalled supernova shock by neutrino heating, Astrophys. J., 295, 14, 1985.

Bionta, R. M., et al. (IMB collaboration): Observation of a neutrino burst in coincidence with supernova 1987A in the Large Magellanic Cloud, Phys. Rev. Lett., 58, 1494, 1987.

Costantini, M. L., Ianni, A., and Vissani, F.: SN1987A and the properties of the neutrino burst, Phys. Rev. D, 70, 043006, 2004.

Costantini, M. L., Ianni, A., and Vissani, F.: Is there a problem with low energy SN1987A neutrinos?, JCAP05, 014, 2007.

Hirata, K., Kajita, T., Koshiba, M., et al.: Observation of a neutrino burst from the supernova SN1987A, Phys. Rev. Lett., 58, 1490, 1987.

Keil, M. T., Raffelt, G. G., and Janka, H. T.: Monte Carlo study of supernova neutrino spectra formation, Astrophys. J., 590, 971, 2003.

Fulgione, W., Molinario, A., and Vigorito, C.: Search for neutrino bursts with LVD at Gran Sasso, $31^{\text {st }}$ ICRC Conf. Proc., Lödz, Poland, 2009. 